\title{
A Two Parameter Lindley Distribution: Its Properties and Applications
}

\author{
Shanker $\mathbf{R}^{1^{*}}$, Kamlesh $\mathbf{K K}^{1}$ and Fesshaye $\mathbf{H}^{2}$ \\ ${ }^{1}$ Department of Statistics, Eritrea Institute of Technology, Eritrea \\ ${ }^{2}$ Department of Economics, College of Business and Economics, Eritrea
}

Submission: March 23, 2017; Published: May 10, 2017

*Corresponding author: Rama S, Department of Statistics, Eritrea Institute of Technology, Eritrea, Email: shankerrama2009@gmail.com

Abstract

In this paper some of the mathematical and statistical properties including mean deviations, order statistics, Bonferroni and Lorenz curves, Renyi entropy measure and stress strength reliability of two-parameter Lindley distribution (TPLD) introduced by Shanker et al. [1] have been discussed. The goodness of fit of TPLD has been discussed with two real lifetime data and the fit has been compared with three parameter generalized Lindley distribution (GLD), two parameter gamma, Weibull and Lognormal distributions and one parameter exponential and Lindley distributions.

Keywords: Mean deviations; Order statistics; Bonferroni and Lorenz curves; Renyi entropy measure; Stress-strength reliability; Goodness of fit

\section{Introduction}

The probability density function (p.d.f.) and the cumulative distribution function (c.d.f.) of Lindley distribution, introduced by Lindley [2] in the context of Bayesian analysis as a counter example of fiducial statistics, are given by

$$
\begin{aligned}
& f(x ; \theta)=\frac{\theta^{2}}{\theta+1}(1+x) e^{-\theta x} ; x>0, \theta>0 \\
& F(x ; \theta)=1-\left[1+\frac{\theta x}{\theta+1}\right] e^{-\theta x} ; x>0, \theta>0
\end{aligned}
$$

The detailed study about its mathematical properties, estimation of parameter and application showing the superiority of Lindley distribution over exponential distribution for the waiting times before service of the bank customers has been done by Ghitany et al. [3]. The Lindley distribution has been generalized, extended and modified by different researchers including Zakerzadeh \& Dolati [4], Nadarajah et al. [5], Deniz \& Ojeda [6], Bakouch et al. [7], Shanker \& Mishra [8,9], Elbatal et al. [10], Ghitany et al. [11], Merovci [12], Liyanage \& Pararai [13], Ashour \& Eltehiwy [14], Oluyede \& Yang [15], Singh et al. [16], Sharma et al. [17], Shanker et al. [18], Alkarni [19], Pararai et al. [20], are some among others. The probability density function (p.d.f.) and cumulative distribution function (c.d.f) of twoparameter Lindley distribution (TPLD) introduced by Shanker et al. [1] are given by

$$
\begin{aligned}
& f(x ; \theta, \alpha)=\frac{\theta^{2}}{\theta+\alpha}(1+\alpha x) e^{-\theta x} ; x>0, \theta>0, \alpha>-\theta \\
& F(x ; \theta, \alpha)=1-\left[\frac{\theta+\alpha+\alpha \theta x}{\theta+\alpha}\right] e^{-\theta x} ; x>0, \theta>0, \alpha>-\theta
\end{aligned}
$$

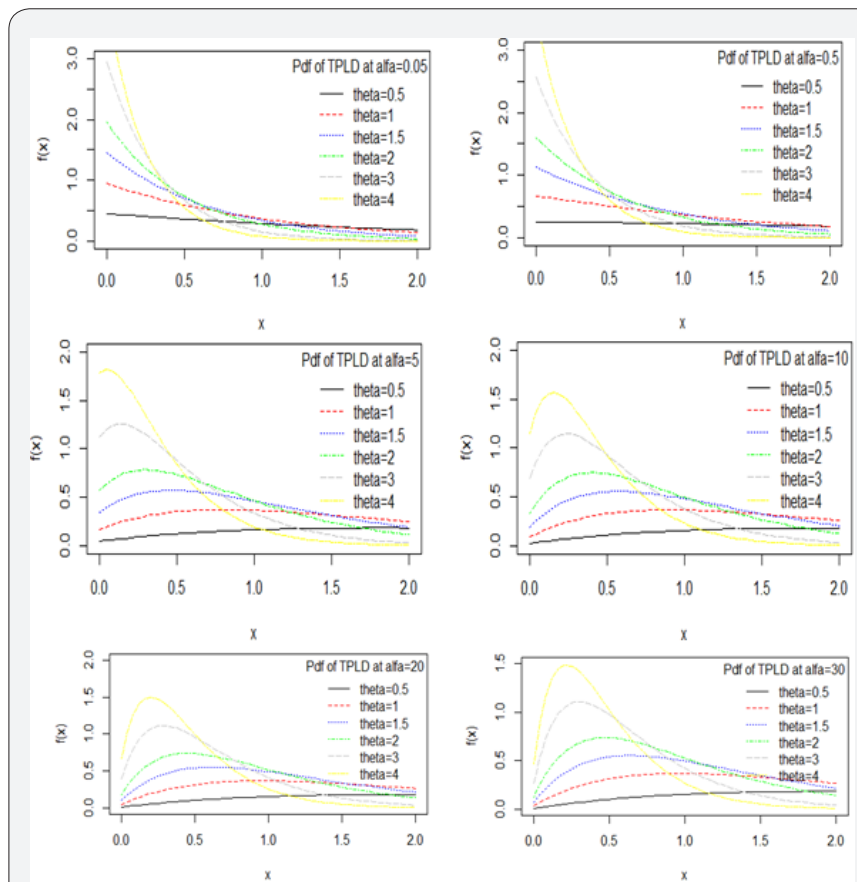

Figure 1:Probability density function of TPLD for varying values of the parameters $\theta$ and $\alpha$. 
At $\alpha=1$, both (1.3) and (1.4) reduce to the corresponding expressions (1.1) and (1.2) of Lindley distribution. The nature and behavior of TPLD (1.3) for varying values of the parameters and have been presented graphically in the Figure 1. It is clear from the graphs of the p.d.f of TPLD that TPLD is always positively skewed.

The th moment about origin of TPLD (1.3) obtained by Shanker et al (2013) is given by

$$
\mu_{r}^{\prime}=\frac{r !(\theta+\alpha+\alpha r)}{\theta^{r}(\theta+\alpha)} ; r=1,2,3, \ldots
$$

It can be easily verified that for $\alpha=1, \mu_{r}^{\prime}$ reduces to the corresponding $\mu_{r}^{\prime}$ of Lindley distribution (1.1).

The first two moments about origin and the variance of TPLD introduced by Shanker et al (2013) are given by

$$
\begin{aligned}
& \mu_{1}^{\prime}=\frac{\theta+2 \alpha}{\theta(\theta+\alpha)} \\
& \mu_{2}^{\prime}=\frac{2(\theta+3 \alpha)}{\theta^{2}(\theta+\alpha)} \\
& \mu_{2}=\frac{\theta^{2}+4 \theta \alpha+2 \alpha^{2}}{\theta^{2}(\theta+\alpha)^{2}}
\end{aligned}
$$

At $\alpha=1$, these moments reduce to the corresponding moments of Lindley distribution. Shanker et al. [1] have derived and discussed some of its statistical and mathematical properties such as shape, moments, coefficient of variation, coefficient of skewness and kurtosis, hazard rate function, mean residual life function and stochastic orderings. They have also discussed the estimation of its parameters using maximum likelihood estimation and method of moments and its goodness of fit over exponential and Lindley distributions. It has been observed that many important mathematical properties of this distribution have not been studied.

In the present paper some of the important properties including mean deviations, order statistics, Bonferroni and Lorenz curves, Renyi entropy measure and stress strength reliability of TPLD of Shanker et al. [1] have been derived and discussed. Its goodness of fit over three parameter generalized Lindley distribution (GLD), two parameter gamma, Weibull and Lognormal distributions and one parameter exponential and Lindley distributions have been illustrated with two real lifetime data sets and found that TPLD gives satisfactory fit.

\section{Mean Deviation about Mean and Median}

The amount of scatter in a population is measured to some extent by the totality of deviations usually from mean and median. These are known as the mean deviation about the mean and the mean deviation about the median defined as
$\delta_{1}(X)=\int_{0}^{\infty}|x-\mu| f(x) d x \quad$ and $\quad \delta_{2}(X)=\int_{0}^{\infty}|x-M| f(x) d x$, respectively, where $\mu=E(X)$ and $M=$ Median $(X)$ The measures $\delta_{1}(X)$ and $\delta_{2}(X)$ can be calculated using the following simplified relationships

$$
\begin{aligned}
& \delta_{1}(X)=\int_{0}^{\mu}(\mu-x) f(x) d x+\int_{\mu}^{\infty}(x-\mu) f(x) d x \\
& =\mu F(\mu)-\int_{0}^{\mu} x f(x) d x-\mu[1-F(\mu)]+\int_{\mu}^{\infty} x f(x) d x \\
& =2 \mu F(\mu)-2 \mu+2 \int_{\mu}^{\infty} x f(x) d x \\
& =2 \mu F(\mu)-2 \int_{0}^{\mu} x f(x) d x
\end{aligned}
$$

and

$$
\begin{aligned}
& \delta_{2}(X)=\int_{0}^{M}(M-x) f(x) d x+\int_{M}^{\infty}(x-M) f(x) d x \\
= & M F(M)-\int_{0}^{M} x f(x) d x-M[1-F(M)]+\int_{M}^{\infty} x f(x) d x \\
= & -\mu+2 \int_{M}^{\infty} x f(x) d x \\
= & \mu-2 \int_{0}^{M} x f(x) d x
\end{aligned}
$$

Using p.d.f. (1.3), and expression for mean of two-parameter Lindley distribution, we have

$$
\begin{aligned}
& \int_{0}^{\mu} x f(x) d x=\mu-\frac{\left\{\theta^{2}\left(\alpha \mu^{2}+\mu\right)+2 \alpha \theta \mu+(2 \alpha+\theta)\right\} e^{-\theta \mu}}{\theta(\theta+\alpha)} \\
& \int_{0}^{M} x f(x) d x=\mu-\frac{\left\{\theta^{2}\left(\alpha M^{2}+M\right)+2 \alpha \theta M+(2 \alpha+\theta)\right\} e^{-\theta M}}{\theta(\theta+\alpha)}
\end{aligned}
$$

Using expressions from (2.1), (2.2),(2.3) and (2.4) and little algebraic simplification, the mean deviation about mean, $\delta_{1}(X)$ and the mean deviation about median, $\delta_{2}(X)$ of TPLD (1.3) are obtained as

$$
\delta_{1}(X)=\frac{2(\alpha \theta \mu+2 \alpha+\theta) e^{-\theta \mu}}{\theta(\theta+\alpha)}
$$

and

$$
\delta_{2}(X)=\frac{2\left\{\theta^{2}\left(\alpha M^{2}+M\right)+2 \alpha \theta M+(2 \alpha+\theta)\right\} e^{-\theta M}}{\theta(\theta+\alpha)}-\mu
$$

It can be easily seen that expressions (3.5) and (3.6) of TPLD (1.3) reduce to the corresponding expressions of Lindley distribution at $\alpha=1$.

\section{Distribution of Order Statistics}

Let $X_{1}, X_{2}, \ldots, X_{n}$ be a random sample of size from twoparameter Lindley distribution (1.3). Let $X_{(1)}<X_{(2)}<\ldots<X_{(n)}$ denote the corresponding order statistics. The p.d.f. and the c.d.f. of the $\mathrm{k}^{\text {th }}$ order statistic, say $Y=X_{(k)}$ are given by 


$$
\begin{aligned}
& f_{Y}(y)=\frac{n !}{(k-1) !(n-k) !} F^{k-1}(y)\{1-F(y)\}^{n-k} f(y) \\
& =\frac{n !}{(k-1) !(n-k) !} \sum_{l=0}^{n-k}\left(\begin{array}{c}
n-k \\
l
\end{array}\right)(-1)^{l} F^{k+l-1}(y) f(y)
\end{aligned}
$$

and

$$
\begin{aligned}
& F_{Y}(y)=\sum_{j=k}^{n}\left(\begin{array}{l}
n \\
j
\end{array}\right) F^{j}(y)\{1-F(y)\}^{n-j} \\
= & \sum_{j=k}^{n} \sum_{l=0}^{n-j}\left(\begin{array}{c}
n \\
j
\end{array}\right)\left(\begin{array}{c}
n-j \\
l
\end{array}\right)(-1)^{l} F^{j+l}(y)
\end{aligned}
$$

respectively, for $k=1,2,3, \ldots, n$.

Thus, the p.d.f. and the c.d.f of the th order statistics of TPLD (1.3) are obtained as

$$
f_{Y}(y)=\frac{n ! \theta^{2}(1+\alpha x) e^{-\theta x}}{(\theta+\alpha)(k-1) !(n-k) !} \sum_{l=0}^{n-k}\left(\begin{array}{c}
n-k \\
l
\end{array}\right)(-1)^{l} \times\left[1-\frac{\theta+\alpha+\alpha \theta x}{\theta+\alpha} e^{-\theta x}\right]^{k+l-1}
$$

and

$$
F_{Y}(y)=\sum_{j=k}^{n} \sum_{l=0}^{n-j}\left(\begin{array}{c}
n \\
j
\end{array}\right)\left(\begin{array}{c}
n-j \\
l
\end{array}\right)(-1)^{l}\left[1-\frac{\theta+\alpha+\alpha \theta x}{\theta+\alpha} e^{-\theta x}\right]^{j+l}
$$

It can be easily verified that the expressions for the p.d.f. and c.d.f. of the th order statistic of TPLD (1.3) reduce to the corresponding expressions for the th order statistic of Lindley distribution at $\alpha=1$.

\section{Bonferroni and Lorenz Curves and Indices}

The Bonferroni and Lorenz curves [22] and Bonferroni and Gini indices have applications not only in economics to study income and poverty, but also in other fields like reliability, demography, insurance and medicine. The Bonferroni and Lorenz curves are defined as

$$
B(p)=\frac{1}{p \mu} \int_{0}^{q} x f(x) d x=\frac{1}{p \mu}\left[\int_{0}^{\infty} x f(x) d x-\int_{q}^{\infty} x f(x) d x\right]=\frac{1}{p \mu}\left[\mu-\int_{q}^{\infty} x f(x) d x\right]
$$

$$
\text { and } L(p)=\frac{1}{\mu} \int_{0}^{q} x f(x) d x=\frac{1}{\mu}\left[\int_{0}^{\infty} x f(x) d x-\int_{q}^{\infty} x f(x) d x\right]=\frac{1}{\mu}\left[\mu-\int_{q}^{\infty} x f(x) d x\right]
$$

Equations (4.1) and (4.2) can also be expressed as

$$
\begin{array}{r}
B(p)=\frac{1}{p \mu} \int_{0}^{p} F^{-1}(x) d x \\
\text { and } L(p)=\frac{1}{\mu} \int_{0}^{p} F^{-1}(x) d x
\end{array}
$$

respectively, where $\mu=E(X)$ and $q=F^{-1}(p)$.
The Bonferroni and Gini indices are thus defined as

$$
B=1-\int_{0}^{1} B(p) d p
$$

and $G=1-2 \int_{0}^{1} L(p) d p$

respectively.

Using p.d.f. (1.3), we get

$\int_{q}^{\infty} x f(x) d x=\frac{\left\{\theta^{2}\left(\alpha q^{2}+q\right)+2 \alpha \theta q+(2 \alpha+\theta)\right\} e^{-\theta q}}{\theta(\theta+\alpha)}$

Now using equation (5.7) in (5.1) and (5.2), we get

$$
B(p)=\frac{1}{p}\left[1-\frac{\left\{\theta^{2}\left(\alpha q^{2}+q\right)+2 \alpha \theta q+(2 \alpha+\theta)\right\} e^{-\theta q}}{\theta+2 \alpha}\right]
$$

and $L(p)=1-\frac{\left\{\theta^{2}\left(\alpha q^{2}+q\right)+2 \alpha \theta q+(2 \alpha+\theta)\right\} e^{-\theta q}}{\theta+2 \alpha}$

Now using equations (5.8) and (5.9) in (5.5) and (5.6), the Bonferroni and Gini indices of TPLD (1.3) are obtained as

$$
\begin{aligned}
& B=1-\frac{\left\{\theta^{2}\left(\alpha q^{2}+q\right)+2 \alpha \theta q+(2 \alpha+\theta)\right\} e^{-\theta q}}{\theta+2 \alpha} \\
& G=-1+\frac{2\left\{\theta^{2}\left(\alpha q^{2}+q\right)+2 \alpha \theta q+(2 \alpha+\theta)\right\} e^{-\theta q}}{\theta+2 \alpha}
\end{aligned}
$$

The Bonferroni and Gini indices of Lindley distribution are particular cases of the Bonferroni and Gini indices (5.10) and (5.11) of TPLD (1.3) for $\alpha=1$.

\section{Renyi Entropy}

An entropy of a random variable $X$ is a measure of variation of uncertainty. A popular entropy measure is Renyi entropy [21]. If $X$ is a continuous random variable having probability density function $f($.$) , then Renyi entropy is defined as$

$$
\begin{aligned}
& T_{R}(\gamma)=\frac{1}{1-\gamma} \log \left\{\int f^{\gamma}(x) d x\right\} \\
& \text { where } . \gamma>0 \text { and } \gamma \neq 1
\end{aligned}
$$

Thus, the Renyi entropy for TPLD (1.3) can be obtained as

$$
\begin{aligned}
& T_{R}(\gamma)=\frac{1}{1-\gamma} \log \left[\int_{0}^{\infty}\left(\frac{\theta^{2}}{\theta+\alpha}\right)^{\gamma}(1+\alpha x)^{\gamma} e^{-\theta \gamma x} d x\right] \\
& =\frac{1}{1-\gamma} \log \left[\int_{0}^{\infty}\left(\frac{\theta^{2}}{\theta+\alpha}\right)^{\gamma} \sum_{j=0}^{\infty}\left(\begin{array}{l}
\gamma \\
j
\end{array}\right)(\alpha x)^{j} e^{-\theta \gamma x} d x\right] \\
& =\frac{1}{1-\gamma} \log \left[\left(\frac{\theta^{2}}{\theta+\alpha}\right)^{\gamma} \sum_{j=0}^{\infty}\left(\begin{array}{l}
\gamma \\
j
\end{array}\right) \alpha^{j} \int_{0}^{\infty} e^{-\theta \gamma x} x^{j+1-1} d x\right] \\
& =\frac{1}{1-\gamma} \log \left[\left(\frac{\theta^{2}}{\theta+\alpha}\right)^{\gamma} \sum_{j=0}^{\infty}\left(\begin{array}{l}
\gamma \\
j
\end{array}\right) \alpha^{j} \frac{\Gamma(j+1)}{(\theta \gamma)^{j+1}}\right]
\end{aligned}
$$


$=\frac{1}{1-\gamma} \log \left[\frac{1}{\theta}\left(\frac{\theta^{2}}{\theta+\alpha}\right)^{\gamma} \sum_{j=0}^{\infty}\left(\begin{array}{l}\gamma \\ j\end{array}\right)\left(\frac{\alpha}{\theta}\right)^{j} \frac{\Gamma(j+1)}{(\gamma)^{j+1}}\right]$

The Renytentropy of Lindley distribution is a particular case of the Renyi entropy of TPLD at $\alpha=1$.

\section{Stress-Strength Reliability}

The stress- strength reliability describes the life of a component which has random strength that is subjected to a random stress. When the stress applied to it exceeds the strength, the component fails instantly and the component will function satisfactorily till $X>Y$. Therefore, $R=P(Y<X)$ is a measure of component reliability and in statistical literature it is known as stress-strength parameter. It has wide applications in almost all areas of knowledge especially in engineering such as structures, deterioration of rocket motors, static fatigue of ceramic components, aging of concrete pressure vessels etc.

Let $X$ and $Y$ be independent strength and stress random variables having TPLD (1.3) with parameter $\left(\theta_{1}, \alpha_{1}\right)$ and $\left(\theta_{2}, \alpha_{2}\right)$ respectively. Then the stress-strength reliability $R$ can be obtained as

$$
\begin{aligned}
& \cdot R=P(Y<X)=\int_{0}^{\infty} P(Y<X \mid X=x) f_{X}(x) d x \\
&=\int_{0}^{\infty} f\left(x ; \theta_{1}, \alpha_{1}\right), F\left(x ; \theta_{2}, \alpha_{2}\right) d x \\
&=1-\frac{\theta_{1}^{2}\left[\alpha_{2} \theta_{1}\left(\theta_{1}+\alpha_{1}\right)+\theta_{2}\left(\theta_{1}+\alpha_{1}\right)\left(\theta_{1}+3 \alpha_{2}\right)+\theta_{2}^{2}\left(2 \theta_{1}+\alpha_{1}+2 \alpha_{2}\right)+\theta_{2}^{3}\right]}{\left(\theta_{1}+\alpha_{1}\right)\left(\theta_{2}+\alpha_{2}\right)\left(\theta_{1}+\theta_{2}\right)^{3}}
\end{aligned}
$$

The expression of stress-strength reliability of Lindley distribution is a particular case of the expression of stressstrength reliability of TPLD (1.3) at . $\alpha_{1}=\alpha_{2}=1$

\section{Maximum likelihood estimate (mle) of parameters}

Let $\left(x_{1}, x_{2}, x_{3}, \ldots, x_{n}\right)$ be a random sample from TPLD (1.3). Let $f_{x}$ be the observed frequency in the sample corresponding to $X=x(x=1,2,3, \ldots k)$ such that $\sum_{x=1}^{k} f_{x}=n$, where is the largest observed value having non-zero frequency. The likelihood function, of TPLD (1.3) is given by

$$
L=\left(\frac{\theta^{2}}{\theta+\alpha}\right)^{n} \prod_{i=1}^{n}(1+\alpha x)^{f_{x}} e^{-n \theta \bar{x}}
$$

The natural log likelihood function is thus obtained as $\ln L=n \ln \theta^{2}-n \ln (\theta+\alpha)+\sum_{x=1}^{k} f_{x} \ln (1+\alpha x)-n \theta \bar{x}$

where $\bar{x}$ is the sample mean.

The maximum likelihood estimate(MLE) $(\hat{\theta}, \hat{\alpha})$ of $(\theta, \alpha)$ of TPLD (1.3) are the solutions of the following equations

$$
\begin{aligned}
& \frac{\partial \ln L}{\partial \theta}=\frac{2 n}{\theta}-\frac{\alpha}{\theta+\alpha}-n \bar{x}=0 \\
& \frac{\partial \ln L}{\partial \alpha}=-\frac{n}{\theta+\alpha}+\sum_{x=1}^{k} \frac{x f_{x}}{1+\alpha x}=0
\end{aligned}
$$

It can be easily seen that equation (8.2.3) gives $\bar{x}=\frac{\alpha \theta+2}{\theta(\alpha \theta+1)}=\mu_{1}^{\prime}$ , mean of TPLD. The equations (8.2.3) and (8.2.4) do not seem to be solved directly. However, Fisher's scoring method can be applied to solve these equations iteratively. We have

$$
\begin{aligned}
& \frac{\partial^{2} \ln L}{\partial \theta^{2}}=-\frac{2 n}{\theta^{2}}+\frac{n}{(\theta+\alpha)^{2}} \\
& \frac{\partial^{2} \ln L}{\partial \theta \partial \alpha}=\frac{n}{(\theta+\alpha)^{2}} \\
& \frac{\partial^{2} \log L}{\partial \alpha^{2}}=\frac{n}{(\theta+\alpha)^{2}}-\sum_{x=1}^{k} \frac{x^{2} f_{x}}{(1+\alpha x)^{2}}
\end{aligned}
$$

The MLE $(\hat{\theta}, \hat{\alpha})$ of $(\theta, \alpha)$ of TPLD (1.3) are the solution of the following equations

$$
\left[\begin{array}{cc}
\frac{\partial^{2} \ln L}{\partial \theta^{2}} & \frac{\partial^{2} \ln L}{\partial \theta \partial \alpha} \\
\frac{\partial^{2} \ln L}{\partial \theta \partial \alpha} & \frac{\partial^{2} \ln L}{\partial \alpha^{2}}
\end{array}\right]_{\substack{\hat{\theta}=\theta_{0} \\
\hat{\alpha}=\alpha_{0}}}\left[\begin{array}{c}
\hat{\theta}=\theta_{0} \\
\hat{\alpha}=\alpha_{0}
\end{array}\right]=\left[\begin{array}{c}
\frac{\partial \ln L}{\partial \theta} \\
\frac{\partial \ln L}{\partial \alpha}
\end{array}\right]_{\hat{\theta}=\theta_{0}}
$$

where $\theta_{0}$ and $\alpha_{0}$ are initial values of as given $\theta$ and $\alpha$ by the method of moments. These equations are solved iteratively till sufficiently close estimates of $\hat{\theta}$ and $\hat{\alpha}$ are obtained. In this paper, R-software has been used to estimate the parameters for the given data sets.

\section{Goodness of Fit}

In this section, we present the goodness of fit of TPLD for some real lifetime data sets and the fit has been compared with three parameter generalized Lindley distribution (GLD), two parameter gamma, Weibull, and Lognormal and one parameter

exponential and Lindley distributions. The probability density functions of the three parameter and two parameter distributions considered in this paper are given in Table 1.

Table 1: Probability density functions of the considered distributions.

\begin{tabular}{|c|c|}
\hline Distribution & Probability Density Function \\
\hline GLD & $f(x ; \theta, \alpha, \beta)=\frac{\theta^{\alpha+1}}{\Gamma(\alpha+1)} \frac{x^{\alpha-1}}{(\beta+\theta)}(\alpha+\beta x) e^{-\theta x} ; x>0, \theta>0, \alpha>0, \beta>0$ \\
\hline
\end{tabular}




\begin{tabular}{|c|c|}
\hline Gamma & $f(x ; \theta, \alpha)=\frac{\theta^{\alpha}}{\Gamma(\alpha)} x^{\alpha-1} e^{-\theta x} ; x>0, \theta>0, \alpha>0$ \\
\hline Weibull & $f(x ; \theta, \alpha)=\alpha \theta x^{\alpha-1} e^{-\theta x^{\alpha}} ; x>0, \theta>0, \alpha>0$ \\
\hline Lognormal & $f(x ; \theta, \alpha)=\frac{1}{\alpha x \sqrt{2 \pi}} e^{-\frac{1}{2}\left(\frac{\log x-\theta}{\alpha}\right)^{2}} ; x>0, \theta>0, \alpha>0$ \\
\hline
\end{tabular}

Table 2: MLE's, -2In L, AIC and K-S Statistics of the fitted distributions of data sets 1 and 2.

\begin{tabular}{|c|c|c|c|c|c|c|c|}
\hline & \multirow[b]{2}{*}{ Model } & \multicolumn{3}{|c|}{ ML Estimates } & \multirow[b]{2}{*}{$-2 \ln L$} & \multirow[b]{2}{*}{ AIC } & \multirow{2}{*}{$\begin{array}{c}\text { K-S } \\
\text { Statistics }\end{array}$} \\
\hline & & $\hat{\theta}$ & $\hat{\alpha}$ & $\hat{\beta}$ & & & \\
\hline \multirow{7}{*}{ Data 1} & TPLD & 0.0622 & 0.1559 & $-\cdots--$ & 128.21 & 132.21 & 0.095 \\
\hline & Gamma & 0.052 & 1.442 & $----\cdot$ & 128.16 & 132.16 & 0.100 \\
\hline & Weibull & 0.034 & 1.306 & ----- & 128.04 & 132.04 & 0.451 \\
\hline & Lognormal & 2.931 & 1.061 & ----- & 131.23 & 135.23 & 0.161 \\
\hline & Lindley & 0.0702 & ----- & ---- & 128.81 & 130.81 & 0.110 \\
\hline & Exponential & 0.0363 & ------ & ---- & 129.47 & 131.47 & 0.156 \\
\hline & GLD & 0.064 & 1.203 & 0.083 & 128.16 & 134.16 & 0.095 \\
\hline
\end{tabular}

\begin{tabular}{|c|c|c|c|}
\hline \multirow{4}{*}{ Data 2 } & TPLD & 0.0108 & 0.1288 \\
\cline { 2 - 4 } & Gamma & 0.010 & 1.794 \\
\cline { 2 - 4 } & Weibull & 0.005 & 1.414 \\
\cline { 2 - 4 } & Lognormal & 4.880 & 0.891 \\
\cline { 2 - 4 } & Lindley & 0.0112 & ----- \\
\cline { 2 - 4 } & Exponential & 0.0056 & $-\cdots---$ \\
\cline { 2 - 4 } & GLD & 0.012 & 1.505 \\
\hline
\end{tabular}

For testing the goodness of fit of TPLD with three parameter generalized Lindley distribution, two parameter Gamma, Weibull and Lognormal distributions and one parameter exponential and Lindley distributions, following lifetime data sets have been considered.

Data Set 1: The following data set represents the failure times (in minutes) for a sample of 15 electronic components in an accelerated life test, Lawless [23]

$\begin{array}{rrrrrrr}19.7 & 1.4 & 5.1 & 6.3 & 10.8 & 12.1 & 18.5 \\ & 22.2 & 23.0 & 30.6 & 37.3 & 46.3 & \\ 53.959 .8 & 66.2 & & & & \end{array}$

Data Set 2: The following data set represents the number of cycles to failure for $25100-\mathrm{cm}$ specimens of yarn, tested at a particular strain level, Lawless [23] Lawless, J.F. (1982): Statistical models and methods for lifetime data, John Wiley and

\begin{tabular}{|c|c|c|c|c|}
\hline Sons ${ }_{-}$New York. & 304.91 & 308.91 & & 0.133 \\
\hline ---- & 304.88 & $30 \$ 88$ & 61 & $0.13 \not 76$ \\
\hline 86 -.--- & 321.89 & 308489 & 157 & 0.697 \\
\hline 175 & 388.17 & $31 \mathrm{1} .98$ & 220 & $0.155^{22}$ \\
\hline 251 & 382.01 & 307.01 & 653 & 0.129 \\
\hline \multicolumn{5}{|c|}{ 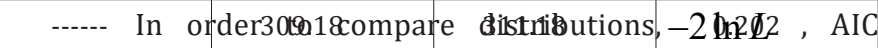 } \\
\hline \multicolumn{5}{|c|}{ 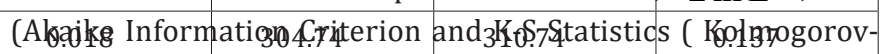 } \\
\hline \multicolumn{5}{|c|}{$\begin{array}{l}\text { Smirnov Statistics) for two real data sets have been computed } \\
\text { and presented in Table } 2 \text {. The formulae for computing AIC } \\
\text { and K-S Statistics are as follows: } A I C=-2 \ln L+2 k \text {, and } \\
K-S=\operatorname{Sup}_{x}\left|F_{n}(x)-F_{0}(x)\right| \text {, where } k=\text { the number of parameters, } \\
n=\text { the sample size and } F_{n}(x) \text { is the empirical distribution } \\
\text { function. The best distribution for modeling lifetime data is the } \\
\text { distribution having lowest value of K-S statistics. }\end{array}$} \\
\hline \multicolumn{5}{|c|}{$\begin{array}{l}\text { It is obvious from the goodness of fit given in table } 2 \text { for } \\
\text { three parameter generalized Lindley distribution (GLD), two } \\
\text { parameter gamma, Weibull, Lognormal distributions and one } \\
\text { parameter exponential and Lindley distributions that TPLD } \\
\text { competes well with these distributions and gives better fit. }\end{array}$} \\
\hline
\end{tabular}

\section{Conclusion}

In the present paper some properties of TPLD including mean deviations, order statistics, Bonferroni and Lorenz curves, Renyi entropy measure and stress strength reliability 
have been derived and discussed. The distribution has been fitted to two real lifetime data sets to test its goodness of fit over three parameter generalized Lindley distribution (GLD), two parameter gamma, Weibull, and Lognormal distributions and one parameter exponential and Lindley distributions. The goodness of fit of TPLD shows that it gives better fit than all the distributions considered and hence TPLD can be considered an important lifetime model for modeling lifetime data over three parameter generalized Lindley distribution (GLD), two parameter gamma, Weibull, and Lognormal distributions and one parameter exponential and Lindley distributions.

\section{References}

1. Shanker R, Sharma S, Shanker R (2013) A two-parameter Lindley distribution for modeling waiting and survival times data. Applied Mathematics 4: 363-368.

2. Lindley DV (1958) Fiducial distributions and Bayes' theorem. Journal of the Royal Statistical Society Series B 20(1): 102-107.

3. Ghitany ME, Atieh B, Nadarajah S (2008) Lindley distribution and its Application. Mathematics Computing and Simulation 78(4): 493-506.

4. Zakerzadeh H, Dolati A (2009) Generalized Lindley distribution. Journal of Mathematical extension 3(2): 13-25.

5. Nadarajah S, Bakouch HS, Tahmasbi R (2011) A generalized Lindley distribution. Sankhya B 73(2): 331-359.

6. Deniz E, Ojeda E (2011) The discrete Lindley distribution-Properties and Applications. Journal of Statistical Computation and Simulation 81: 1405-1416.

7. Bakouch HS, Al-Zaharani B, Al-Shomrani A, Marchi V, Louzad F (2012) An extended Lindley distribution. Journal of the Korean Statistical Society 41(1): 75-85.

8. Shanker R, Mishra A (2013) A two-parameter Lindley distribution. Statistics in Transition-new series 14 (1): 45-56.

9. Shanker R, Mishra A (2013) A quasi Lindley distribution. African Journal of Mathematics and Computer Science Research 6(4): 64-71.

10. Elbatal I, Merovi F, Elgarhy M (2013) A new generalized Lindley distribution. Mathematical theory and Modeling 3(13): 30-47.
11. Ghitany M, Al-Mutairi D, Balakrishnan N, Al-Enezi I (2013) Power Lindley distribution and associated inference. Computational Statistics and Data Analysis 64: 20-33.

12. Merovci F (2013) Transmuted Lindley distribution. Int J Open Problems Compt Math 6(2): 63-72.

13. Liyanage GW, Pararai M (2014) A generalized Power Lindley distribution with applications. Asian journal of Mathematics and Applications 2014: 1-23.

14. Ashour S, Eltehiwy MA (2015) Exponentiated Power Lindley distribution. J Adv Res 6(6): 895-905.

15. Oluyede BO, Yang T (2014) A new class of generalized Lindley distribution with applications. Journal of Statistical Computation and Simulation 85(10): 2072-2100.

16. Singh SK, Singh U, Sharma VK (2014) The Truncated Lindley distribution- inference and Application. J Stat Appl Pro 3(2): 219-228.

17. Sharma V, Singh S, Singh U, Agiwal V (2015) The inverse Lindley distribution- A stress-strength reliability model with applications to head and neck cancer data. Journal of Industrial \&Production Engineering 32(3): 162-173.

18. Shanker R, Hagos F, Sujatha S (2015) On modeling of Lifetimes data using exponential and Lindley distributions. Biometrics \& Biostatistics International Journal 2(5): 1-9.

19. Alkarni S (2015) Extended Power Lindley distribution-A new Statistical model for non-monotone survival data. European journal of statistics and probability 3(3): 19-34.

20. Pararai M, Liyanage GW, Oluyede BO (2015) A new class of generalized Power Lindley distribution with applications to lifetime data. Theoretical Mathematics \& Applications 5(1): 53-96.

21. Renyi A (1961) On measures of entropy and information, in proceedings of the $4^{\text {th }}$ Berkeley symposiumon Mathematical Statistics and Probability, Berkeley, Volume 1, University of California press, USA, pp. 547-561.

22. Bonferroni CE (1930) Elements of General Statistics. In: Seeber (Ed.), Firenze, Italy.

23. Lawless JF (1982) Statistical models and methods for lifetime data. In: John Wiley \& Sons (Ed.), New York, USA.

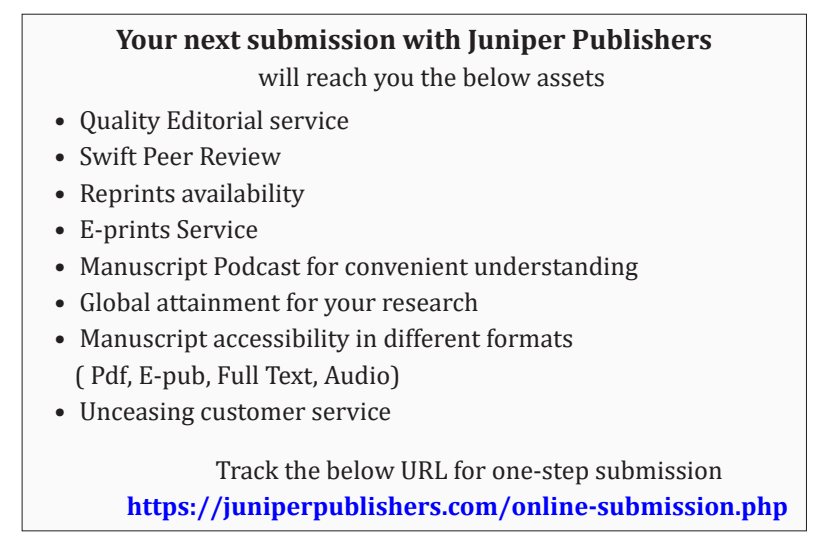

